\title{
Clinical significance and effect of IncRNA BBOX1-AS1 on the proliferation and migration of lung squamous cell carcinoma
}

\author{
YU ZHANG $^{1}$, XIAO WANG ${ }^{2}$, XIAN-KUI CHENG ${ }^{1}$, YUAN-YUAN ZONG $^{1}$, \\ RONG-QUAN HE ${ }^{3}$, GANG CHEN ${ }^{4}$ and YE-JUN QIN ${ }^{1}$ \\ ${ }^{1}$ Department of Pathology, Shandong Provincial Hospital Affiliated to Shandong First Medical University; \\ ${ }^{2}$ Department of Orthopedics, Shandong Second Provincial General Hospital, Shandong Provincial ENT Hospital, \\ Jinan, Shandong 250000; Departments of ${ }^{3}$ Oncology and ${ }^{4}$ Pathology, The First Affiliated Hospital of \\ Guangxi Medical University, Nanning, Guangxi Zhuang Autonomous Region 530021, P.R. China
}

Received July 29, 2021; Accepted October 19, 2021

DOI: $10.3892 / \mathrm{ol} .2021 .13135$

\begin{abstract}
Long non-coding RNAs (lncRNAs) have a role in the occurrence and development of lung squamous cell carcinoma (LUSC). IncRNA $\gamma$-butyrobetaine hydroxylase 1 (BBOX1)-antisense 1 (AS1) may contribute to disease development. However, there are no studies on the role of BBOX1-AS1 in LUSC to date. In the present study, an in-house gene microarray analysis was performed to detect the differentially expressed lncRNAs and mRNAs between three pairs of LUSC and normal lung tissues. Only one lncRNA, BBOX1-AS1, was differentially expressed in the in-house microarray and The Cancer Genome Atlas (TCGA), Gene Expression Omnibus (GEO) and ArrayExpress databases. Reverse transcription-quantitative PCR (RT-qPCR)
\end{abstract}

Correspondence to: Professor Dr Gang Chen, Department of Pathology, The First Affiliated Hospital of Guangxi Medical University, 6 Shuangyong Road, Nanning, Guangxi Zhuang Autonomous Region 530021, P.R. China

E-mail: chengang@gxmu.edu.cn

Professor Dr Ye-Jun Qin, Department of Pathology, Shandong Provincial Hospital Affiliated to Shandong First Medical University, 324 Jingwu Road, Jinan, Shandong 250000, P.R. China

E-mail: qinyejun@163.com

Abbreviations: lncRNA, long noncoding RNA; NSCLC, non-small cell lung cancer; LUSC, lung squamous cell carcinoma; RT-qPCR, reverse transcription-quantitative PCR; GEO, Gene Expression Omnibus; TCGA, The Cancer Genome Atlas; GO, Gene Ontology; KEGG, Kyoto Encyclopedia of Genes and Genomes; ROC, receiver operating characteristic; $\mathrm{BP}$, biological process; $\mathrm{CC}$, cellular component; MF, molecular function; DAVID, Database for Annotation, Visualization and Integrated Discovery; STRING, Search Tool for the Retrieval of Interacting Genes; NCBI, National Center of Biotechnology Information; SROC, summary receiver operating characteristic; SRA, Sequence Read Archive; siRNA, small interfering RNA; SMD, standard mean deviation

Key words: BBOX1-AS1, LUSC, RT-qPCR, proliferation, migration was then performed and the original RNA-sequencing data from the TCGA, GEO and ArrayExpress datasets were used to determine the expression and clinical value of BBOX1-AS1 in LUSC. In addition, a Cell Counting Kit-8 assay, cell cycle analysis and scratch assay were performed to explore whether BBOX1-AS1 expression affected the proliferation and migration of LUSC cells in vitro. The results of the RT-qPCR analysis and data obtained from the TCGA database, GEO datasets, in-house gene microarray and standard mean deviation analysis all supported the upregulated expression level of BBOX1-AS1 in LUSC. Furthermore, silencing of BBOX1-AS1 inhibited the proliferation and migration of LUSC cells according to in vitro assays. In addition, the cells were arrested in S-phase after knockdown of BBOX1-AS1. In conclusion, the expression level of BBOX1-AS1 was upregulated in LUSC tissues. BBOX1-AS1 may exert an oncogenic effect on LUSC by regulating various biological functions. However, additional functional experiments should be performed to verify the exact mechanism.

\section{Introduction}

Lung cancer is one of the most common and lethal cancer types with a high rate of mortality worldwide $(1,2)$. Lung cancer accounts for $14 \%$ of new cancer cases and has a $26 \%$ mortality rate in males; for females, it accounts for $13 \%$ of new cancer cases and has a $25 \%$ mortality rate (3). Non-small-cell lung cancer (NSCLC) is the primary subtype of lung cancer and is composed of two major histologic groups: Lung squamous cell carcinoma (LUSC) and lung adenocarcinoma. Treatment strategies, including surgical resection, radiotherapy and chemotherapy, have developed rapidly in recent years, but the overall survival of patients with lung cancer remains poor $(4,5)$. Biomarkers that may be used to detect or diagnose LUSC, improve the survival rate and reduce recurrence are lacking (6). Therefore, it is necessary to develop novel therapeutic strategies for LUSC.

Long non-coding RNAs (lncRNAs) range from 200 nucleotides to $100 \mathrm{~kb}$ in length and are a class of non-protein-coding RNAs $(7,8)$. IncRNAs are known to have various roles in disease development, regulation of metabolism, epigenetic 
gene control and transcriptional regulation $(9,10)$. Over the past decade, lncRNAs have been determined to be widely involved in the proliferation, apoptosis, invasion and metastasis of malignancies $(11,12)$. Increasing evidence in lung cancer has indicated that dysregulated lncRNAs may affect the development and occurrence of lung cancer by modifying the biological functions and self-renewal abilities of cancer cells (12-14). Furthermore, it was reported that dysregulated lncRNAs have a role in the drug resistance of lung cancer $(15,16)$.

lncRNA $\gamma$-butyrobetaine hydroxylase 1 (BBOX1)-antisense 1 (AS1) [National Center of Biotechnology Information (NCBI) Gene ID: 103695435] is located on chromosome 11p14.2-p14.1. As previously reported, BBOX1-AS1 has a role in the development of various diseases, including NSCLC, colorectal cancer, cervical cancer and gastric cancer (17-20). BBOX1-AS1 was overexpressed in NSCLC and in colorectal, cervical and gastric cancers. BBOX1-AS1 may also facilitate the proliferation, invasion and migration of these cancer cells (17-20). However, the functions of BBOX1-AS1 in LUSC have yet to be studied independently.

In the present study, the clinical significance and effect of the differential expression of BBOX1-AS1 in LUSC was investigated. In vitro assays and in silico analyses were performed to examine the biological functions and probable mechanisms of BBOX1-AS1 in LUSC. A flow diagram of the present study is presented in Fig. 1.

\section{Materials and methods}

Patient tissue samples. A total of three pairs of LUSC tissues and normal lung tissues obtained $>5 \mathrm{~cm}$ from the edge of the LUSC lesion were collected between August 2017 and October 2017 from the Department of Pathology of the First Affiliated Hospital of the Guangxi Medical University (Nanning, China). The patients' age ranged from 51 to 58 years. All three patients were definitively diagnosed with LUSC and underwent surgical resection without any further treatment. The pathological diagnoses were independently performed by at least two blinded pathologists. All methods used adhered to the relevant guidelines and were approved by the Ethical Committee of the First Affiliated Hospital of Guangxi Medical University (Nanning, China). Consent forms for the use of tissues were signed by the doctors and patients involved in the study.

In-house microarray analysis. An in-house microarray analysis was performed to detect differentially expressed lncRNAs between LUSC and normal lung tissues. Microarray hybridization and sample analysis were performed by Kangchen Biotech. The detailed methods were performed as previously described by Zhang et al (21). The significantly differentially expressed lncRNAs between LUSC and normal lung tissues were selected using the criteria of Ifold changel $\geq 2$ and $\mathrm{P} \leq 0.05$. Similarly, the differentially expressed mRNAs were identified using microarray analysis.

Validation of the expression and clinical role of BBOX1-AS1. Expression analysis in clinical samples was performed using the original RNA sequencing (RNA-seq) data from The
Cancer Genome Atlas (TCGA, cancergenome.nih.gov) database to determine BBOX1-AS1 expression between LUSC and noncancerous lung tissues. The LUSC cohort comprised 502 LUSC cases and 49 corresponding noncancerous lung samples. The original expression data were normalized using the $\mathrm{R}$ package DESeq. Differences in the expression of BBOX1-AS1 between LUSC tissues and normal lung tissues were identified from the TCGA dataset $(22,23)$. The relationships of BBOX-AS1 expression and clinicopathological parameters (age, sex, ethnicity, tumor stage, regional lymph nodes, distant metastasis and stage) were determined from the TCGA data. A receiver operating characteristic (ROC) curve was created to determine the clinical value of BBOX1-AS1 expression in distinguishing normal and LUAD tissue. Gene Expression Omnibus (GEO, http://www.ncbi.nlm.nih. gov/geo/) data and the ArrayExpress (ebi.ac.uk/arrayexpress/) database were used to verify BBOX1-AS1 expression in LUSC. Kaplan-Meier curve analysis was used to assess BBOX1-AS1 expression and the 5-year survival rates of patients with LUSC with an endpoint of death or the end of the study period.

The LUSC-related BBOX1-AS1 microarray and RNA-seq datasets were downloaded from GEO, ArrayExpress, the TCGA, Sequence Read Archive (SRA, https://trace.ncbi.nlm. nih.gov/Traces/sra/) and Oncomine (https://www.oncomine. org/). Publications related to BBOX1-AS1 in LUSC were also selected from 12 online databases: PubMed, EMBASE, Web of Science, Science Direct, Cochrane Central Register of Controlled Trials, Google Scholar, Ovid, Chinese CNKI, LILACS, Wiley Online Library, Wan Fang, Chong Qing VIP and China Biology Medicine disc. The retrieval date was set to September 15, 2020, with the following Boolean search terms: (lung OR pulmonary OR 'NSCLC' OR 'LC') AND ('IncRNA' OR 'noncoding RNA' OR 'noncoding RNA' OR 'noncoding RNA' OR gene). Two different investigators ( $\mathrm{YZ}$ and $\mathrm{XW}$ ) cross-checked the literature. A group discussion was used to resolve any disagreement. The number of false positives, true positives, false negatives and true negatives was extracted as previously reported $(24,25)$. The standard mean deviation (SMD) and summary receiver operating characteristic (SROC) curve of BBOX1-AS1 in LUSC were presented in Stata 14.0 (Stata Corp.). A fixed-effects model was used to analyze the pooled SMD to determine the expression of BBOX1-AS1 in LUSC compared to that in the normal group. The funnel plot used to evaluate the publication bias. The asymmetry of the funnel plot could display the validity of conclusions.

Cell culture and transfection with small interfering (si) $R N A$. Three human LUSC cell lines (NCI-H1703, NCI-H226 and SK-MES-1) and human bronchial epithelial cells (BEAS-2B) were obtained from the American Type Culture Collection. These four cell lines were cultured in DMEM with $10 \%$ heat-inactivated fetal bovine serum (Invitrogen; Thermo Fisher Scientific, Inc.). All of the cells were incubated in a humidified atmosphere with $5 \% \mathrm{CO}_{2}$ at $37^{\circ} \mathrm{C}$.

RNA interference (RNAi) was performed to determine the underlying effect of BBOX1-AS1 on biological processes in LUSC. The lentiviral vector containing siRNA targeting BBOX1-AS1 was synthesized by GeneChem (sense, 5'-CTGCTTTGCTCTTCAGACTTATT-3'; antisense, 5'-TAAGTCTGAAGAGCAAAGCAG-3'). The siRNA vectors 


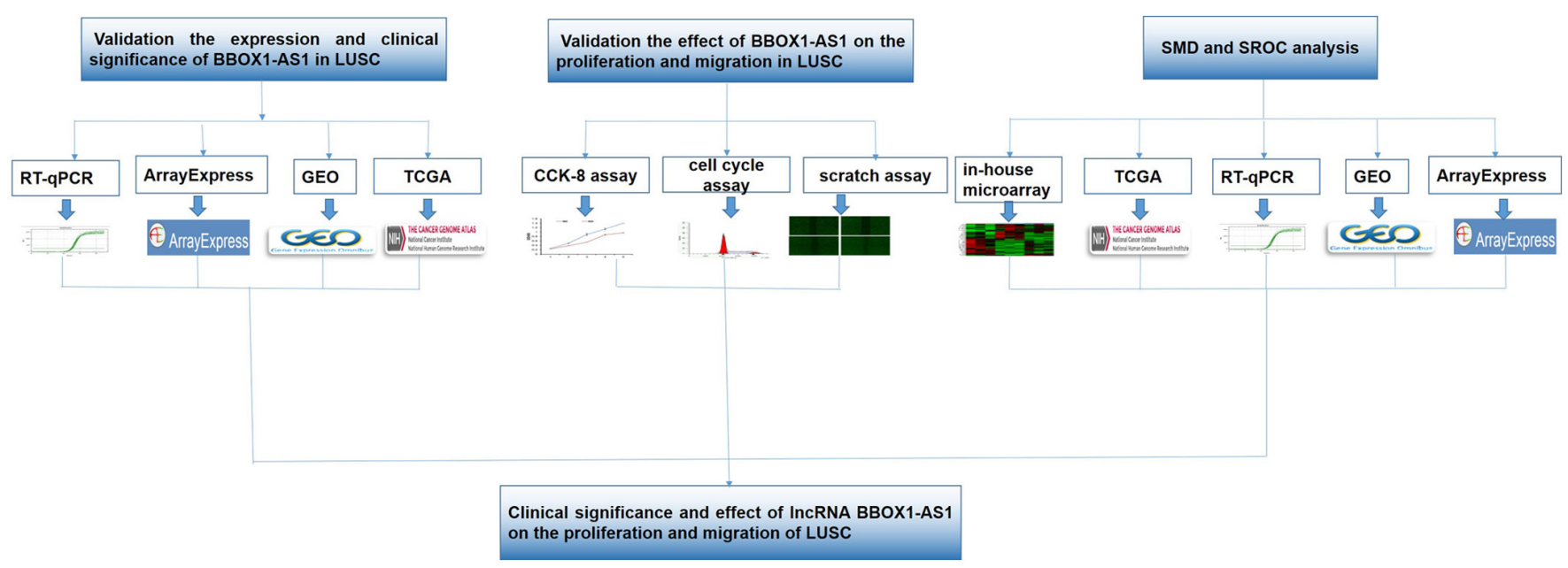

Figure 1. Flow chart of this study. LUSC, lung squamous cell carcinoma; lncRNA, long non-coding RNA; BBOX1-AS1, $\gamma$-butyrobetaine hydroxylase 1 antisense 1; CCK-8, Cell Counting Kit-8; SMD, standard mean deviation; SROC, summary receiver operating characteristic; TCGA, The Cancer Genome Atlas; GEO Gene Expression Omnibus; RT-qPCR, reverse transcription-quantitative PCR.
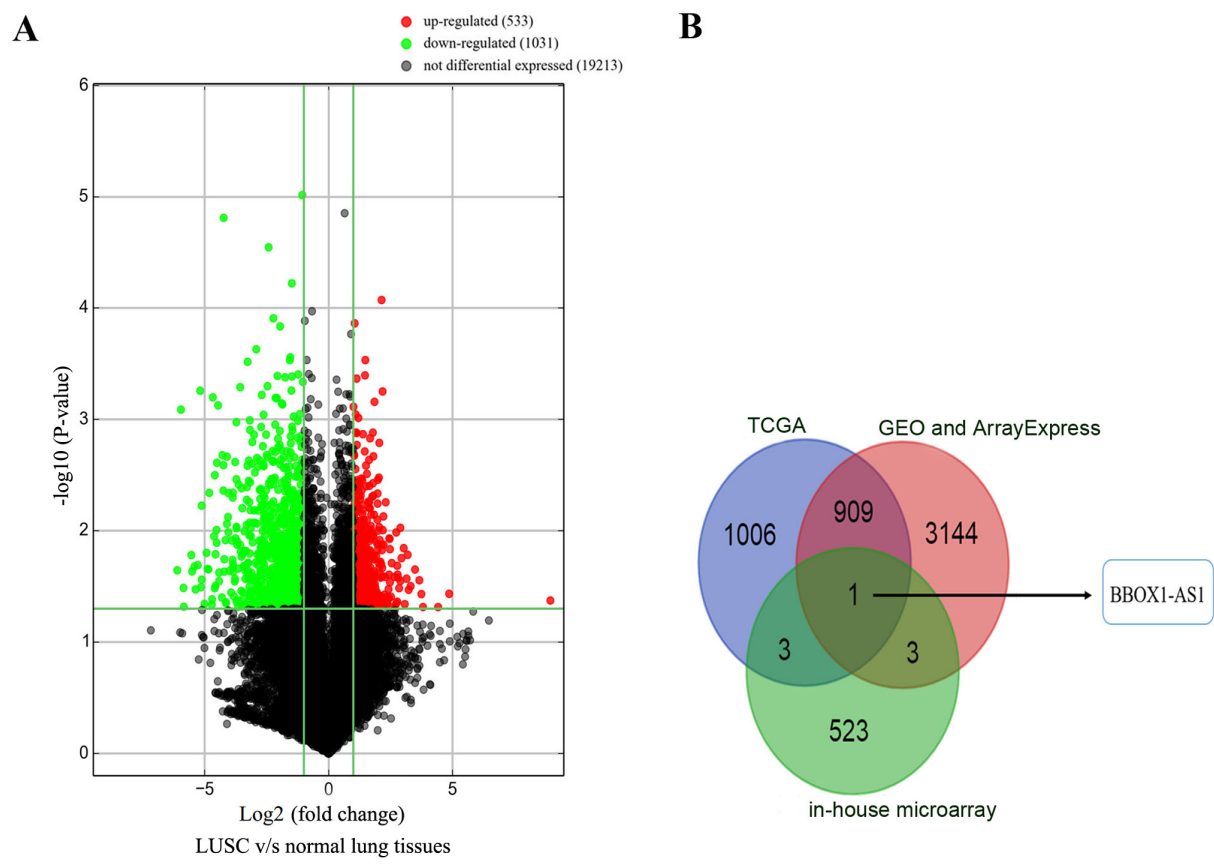

Figure 2. In-house microarray of lncRNAs in LUSC tissue. (A) Volcano plot; (B) the overlapping upregulated lncRNAs based on the datasets from the TCGA, GEO and ArrayExpress databases and the in-house microarray. TCGA, The Cancer Genome Atlas; GEO Gene Expression; LUSC, lung squamous cell carcinoma; lncRNA, long non-coding RNA; BBOX1-AS1, $\gamma$-butyrobetaine hydroxylase 1 antisense 1.

used to knock down the BBOX1-AS1 gene (KD) and the negative siRNA controls (NC; cat. no. CON077, GeneChem) were transfected into the NCI-H1703 and SK-MES-1 cell lines using Lipofectamine $2000^{\circledR}$ (Thermo Fisher Scientific, Inc.) when the cells reached $80 \%$ confluency. Puromycin ( $2 \mu \mathrm{g} / \mathrm{ml}$; Corning, Inc.; cat. no. 21-031-CVR) was applied for filtering the stable cell lines at $48 \mathrm{~h}$ after transfection. A fluorescence microscope was used to observe cell growth after transfection.

Reverse transcription-quantitative PCR (RT-qPCR). Total RNA from NCI-H1703, NCI-H266, SK-MES-1 and BEAS-2B cells was isolated using TRIzol reagent (Shanghai Pufei Biotech
Co., Ltd.) and reverse-transcribed using a Promega M-MLV Kit (Shanghai Pufei Biotech Co., Ltd.). qPCR was performed using the LightCycler 480 Real-time PCR System (Roche). The specific primers were synthesized as follows: BBOX1-AS1 forward, 5'-GATGGGCACATTTGGAAGTT-3' and reverse, 5'-CAGCGTTAGGTTTGGAGTTG-3'; and GAPDH (internal control) forward, 5'-TGACTTCAACAGCGACACCCA-3' and reverse, 5'-CACCCTGTTGCTGTAGCCAAA-3'. All experiments were performed in triplicate. The levels of BBOX1-AS1 expression were standardized to those of GAPDH and calculated using the delta $\mathrm{Cq}$ method (26). Furthermore, the transfection efficiency of the lentiviral vector containing BBOX1-AS1 siRNA was detected via RT-qPCR. 
Cell Counting Kit-8 (CCK-8) assay. A CCK-8 assay was performed to examine the proliferation of LUSC cells in two different groups (KD group and NC group). Cell viability was determined with a CCK-8 (Beyotime Institute of Biotechnology) following the manufacturer's instructions. The detailed methods were performed as previously described by Zhang et al (27). The viability of NCI-H1703 and SK-MES-1 cells at days one, two, three, four and five was assessed and the absorbance at $450 \mathrm{~nm}$ was read using a microplate reader (Infinite M200 Micro Plate Reader; Tecan Group). The fold change in the optical density at $450 \mathrm{~nm}$ was considered to represent changes in cell viability. Experiments were performed in triplicate.

Flow cytometric cell cycle analysis. Flow cytometry was used to measure the effect of BBOX1-AS1 on the cell cycle distribution. The detailed method of the flow cytometric cell cycle assay was previously described by Zhang et al (27). Cells in the different phases of the cell cycle (G1, S and G2) were identified using FACSCalibur (EMD Millipore) and data analysis was performed using ModFit 3.2.1 (Verity Software House, Inc.). Each test was performed in triplicate.

Scratch assay. A scratch assay was performed to observe the potential effect of BBOX1-AS1-siRNA on the migration of LUSC cells. The detailed method of the scratch assay was performed as previously described by Zhang et al $(27,28)$. Several random fields of the monolayer were captured directly under a microscope at 0 and $24 \mathrm{~h}$ after scratching. All experiments were performed in triplicate.

Potential pathways and functions associated with BBOX1-AS1. Genes associated with BBOX1-AS1 in LUSC were subjected to Gene Ontology (GO) and Kyoto Encyclopedia of Genes and Genomes (KEGG) analyses to determine fundamental biological functions and downstream pathways (29-31). The genes associated with BBOX1-AS1 in the GEO datasets were determined using the Psych R programming package. The co-expressed genes in the TCGA database overlapping with the upregulated mRNAs in the in-house microarray for bioinformatics analysis were selected. The Database for Annotation, Visualization and Integrated Discovery (DAVID; http://david.abcc.ncifcrf.gov/) website was used for GO and KEGG analyses. The functional terms in the categories biological process (BP), cellular component (CC) and molecular function (MF) were obtained from the GO analysis and the GO functional network was drawn using Cytoscape (v3.5.1, http://cytoscape.org).

The interaction pairs of the co-expressed genes were determined using the Search Tool for the Retrieval of Interacting Genes (STRING; version 9.0; http://string-db.org) (32). STRING provides a global database for numerous organisms and the predicted and known interactions were scored. The interactional pairs in the protein-protein interaction (PPI) network were nominated based on a combined score of $>0.4$.

Fluorescence in situ hybridization (FISH). The FISH assay was performed to determine the localization of BBOX1-AS1 in the nucleus or cytoplasm of LUSC tissues. Cy3-labeled BBOX1-AS1 probes were designed and synthesized by General Biol. Hybridization was performed overnight with BBOX1-AS1 probes according to the manufacturer's instructions. Images
Table I. Correlation between BBOX1-AS1 expression and clinicopathological features based on TCGA database.

\begin{tabular}{|c|c|c|c|c|}
\hline \multirow{2}{*}{$\begin{array}{l}\text { Clinicopathological } \\
\text { feature }\end{array}$} & \multirow[b]{2}{*}{$\mathrm{N}$} & \multicolumn{3}{|c|}{ BBOX1-AS1 expression } \\
\hline & & Mean \pm SD & $\mathrm{R}$ & P-value \\
\hline Age, years & & & 0.05 & 0.272 \\
\hline$<60$ & 91 & $5.752 \pm 3.939$ & & \\
\hline$\geq 60$ & 401 & $5.260 \pm 3.838$ & & \\
\hline Sex & & & 0.054 & 0.230 \\
\hline Male & 373 & $5.243 \pm 3.951$ & & \\
\hline Female & 128 & $5.723 \pm 3.736$ & & \\
\hline Ethnicity & & & 0.077 & 0.004 \\
\hline White & 348 & $5.219 \pm 3.533$ & & \\
\hline Black & 31 & $5.777 \pm 4.309$ & & \\
\hline Asian & 9 & $9.209 \pm 4.086$ & & \\
\hline T stage & & & 0.019 & 0.674 \\
\hline $\mathrm{T} 1+\mathrm{T} 2$ & 406 & $5.330 \pm 3.637$ & & \\
\hline $\mathrm{T} 3+\mathrm{T} 4$ & 95 & $5.518 \pm 4.883$ & & \\
\hline N stage & & & 0.135 & 0.140 \\
\hline N0-N1 & 280 & $5.096 \pm 3.376$ & & \\
\hline N2-N3 & 37 & $6.721 \pm 6.450$ & & \\
\hline M stage & & & 0.006 & 0.919 \\
\hline M0 & 321 & $5.134 \pm 3.567$ & & \\
\hline M1 & 6 & $5.286 \pm 3.639$ & & \\
\hline Stage & & & 0.045 & 0.318 \\
\hline $\mathrm{I}+\mathrm{II}$ & 408 & $5.310 \pm 3.641$ & & \\
\hline III+IV & 89 & $5.766 \pm 4.924$ & & \\
\hline
\end{tabular}

$\mathrm{T}$, primary tumor; $\mathrm{N}$, regional lymph nodes; $\mathrm{M}$, distant metastasis; $\mathrm{SD}$, standard deviation; BBOX1-AS1, $\gamma$-butyrobetaine hydroxylase 1 antisense 1 .

were collected using a Leica DM 4000B laser scanning confocal microscope (Leica Instrument, Inc.). The sequence of the BBOX1-AS1 probe for FISH was CAGGGTAACCGTAG CATGACCTAGAAATAGTCCTCTCA. All experiments were performed at least three times.

Statistical analysis. Statistical analysis was performed using SPSS v22.0 (IBM Corporation). Student's t-test was used to compare the differences between the two groups and the Kruskal-Wallis H-test was employed to evaluate differences among $>2$ groups. Spearman's rank correlation was utilized to evaluate the correlation between BBOX1-AS1 expression and clinicopathological features. A ROC curve was plotted to distinguish LUSC from normal lung tissues by gene expression. A two-sided $\mathrm{P}<0.05$ was considered to indicate statistical significance.

\section{Results}

Differentially expressed lncRNAs via in-house microarray analysis. Based on the in-house microarray analysis, a total 


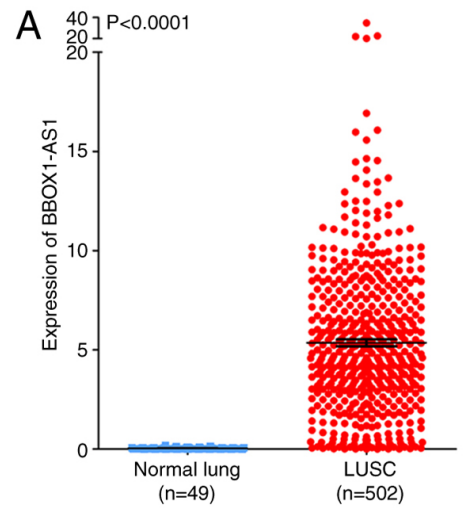

D
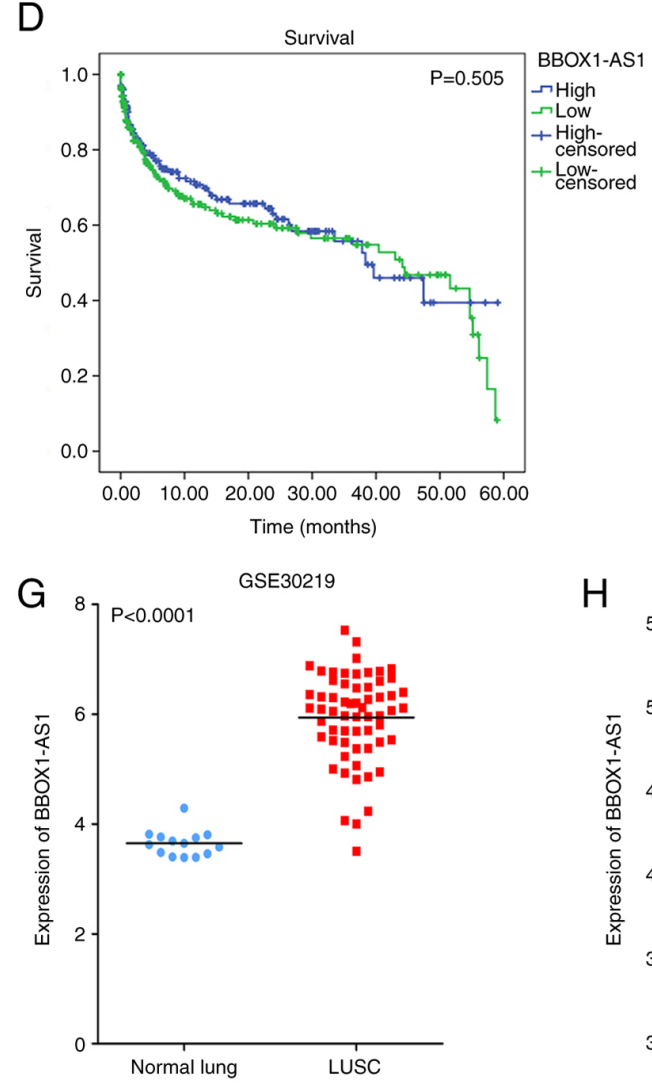

B
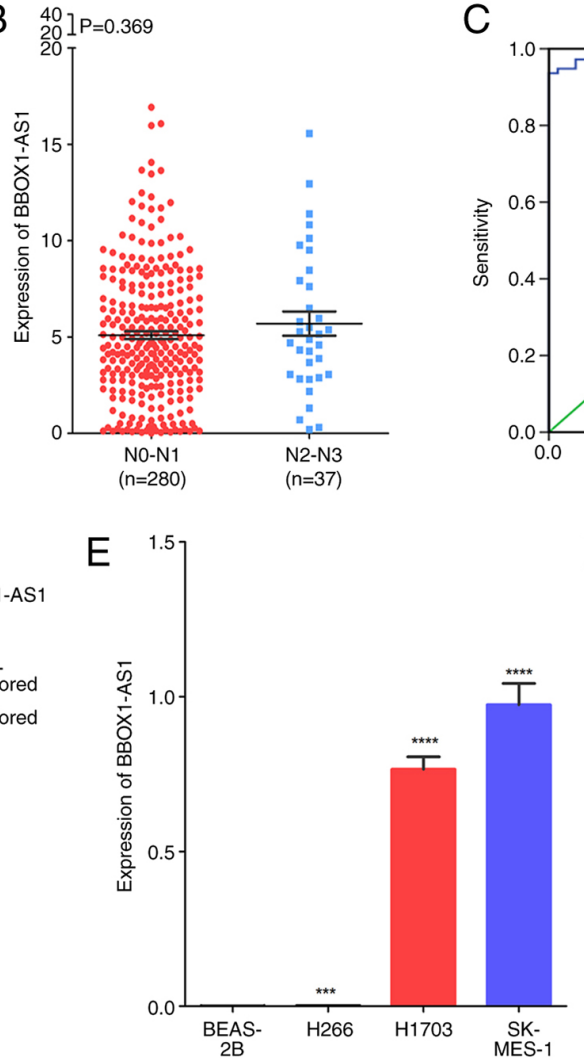

$\mathrm{H}$

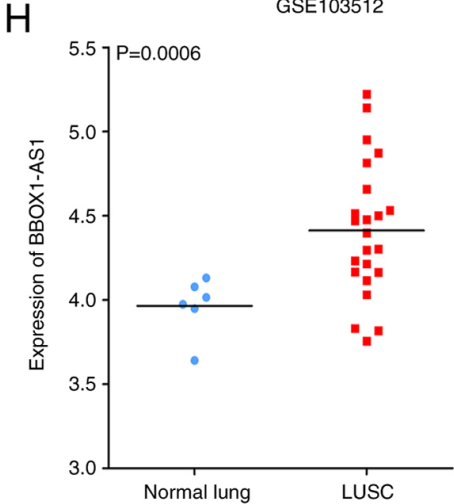

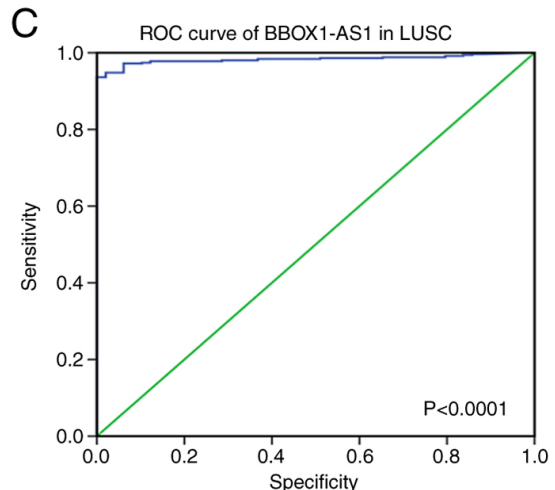

$\mathrm{F}$
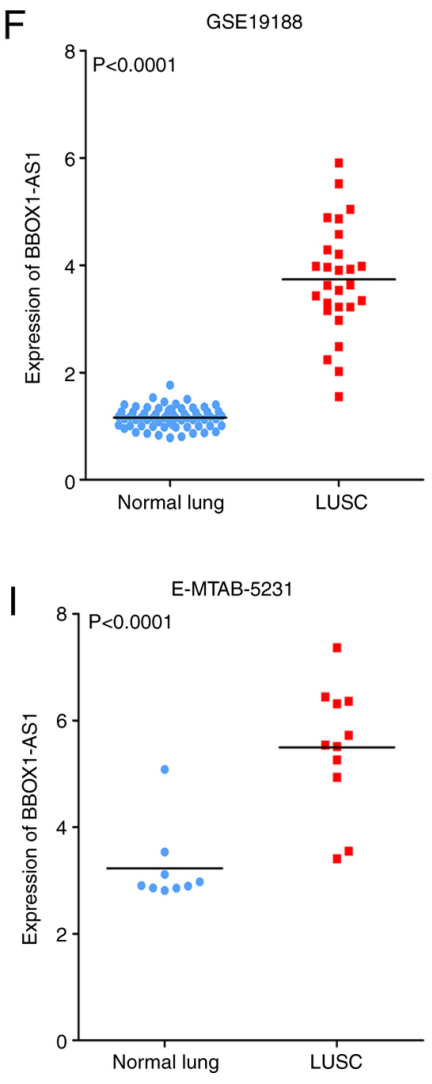

Figure 3. Clinical significance of BBOX1-AS1 in LUSC based on RT-qPCR, as well as TCGA, GEO and ArrayExpress datasets. (A) Differential expression of BBOX1-AS1 between LUSC and normal lung tissue based on the TCGA dataset. (B) Differential expression of BBOX1-AS1 in the lymphatic metastasis group vs. no lymphatic metastasis group based on the TCGA dataset. (C) ROC curve of BBOX1-AS1 to discriminate LUSC from normal tissues. (D) Kaplan-Meier survival curves for BBOX1-AS1 expression in LUSC. (E) Differential expression of BBOX1-AS1 in LUSC cell lines based on RT-qPCR; ${ }^{* * * *} \mathrm{P}<0.001,{ }^{* * * *} \mathrm{P}<0.0001$ vs. BEAS-2B. (F-I) Differential expression of BBOX1-AS1 between LUSC and normal lung tissue in GEO and ArrayExpress datasets, including the (F) GSE19188 profile, (G) GSE30219 profile, (H) GSE103512 profile, and (I) E-MTAB-5231 profile. LUSC, lung squamous cell carcinoma; BBOX1-AS1, $\gamma$-butyrobetaine hydroxylase 1 antisense 1; ROC, receiver operating characteristic; TCGA, The Cancer Genome Atlas; GEO Gene Expression Omnibus; RT-qPCR, reverse transcription-quantitative PCR.

of 20,777 lncRNAs were identified. Only 533 upregulated lncRNAs and 1,031 downregulated lncRNAs were significantly differentially expressed between LUSC and normal lung tissues (Ifold changel $\geq 2, \mathrm{P} \leq 0.05$; Fig. 2A). The upregulated lncRNAs were extracted from the TCGA, GEO and ArrayExpress databases. Only one upregulated lncRNA, BBOX1-AS1, overlapped among the TCGA, GEO, ArrayExpress and in-house microarray data (Fig. 2B).

Clinical significance of BBOX1-AS1 in LUSC. Increased relative expression of BBOX1-AS1 was observed in LUSC tissues $(5.358 \pm 0.174)$ compared with that in noncancerous tissues based on TCGA $(0.042 \pm 0.006, \mathrm{P}<0.0001$; Fig. 3A). High BBOX1-AS1 expression was related to the clinicopathological characteristic of lymphatic metastasis $(\mathrm{P}<0.05$; Fig. 3B; Table I). No statistical significance was obtained for any of the other clinicopathological parameters in the TCGA database. Furthermore, the AUC of BBOX1-AS1 was 0.983 (95\% CI: 0.973-0.993, P<0.0001; Fig. 3C), which indicated a high possibility that BBOX1-AS1 was able to discriminate LUSC from normal lung tissues. A trend that patients with low BBOX1-AS1 expression survived longer than patients 
$\mathbf{A}$

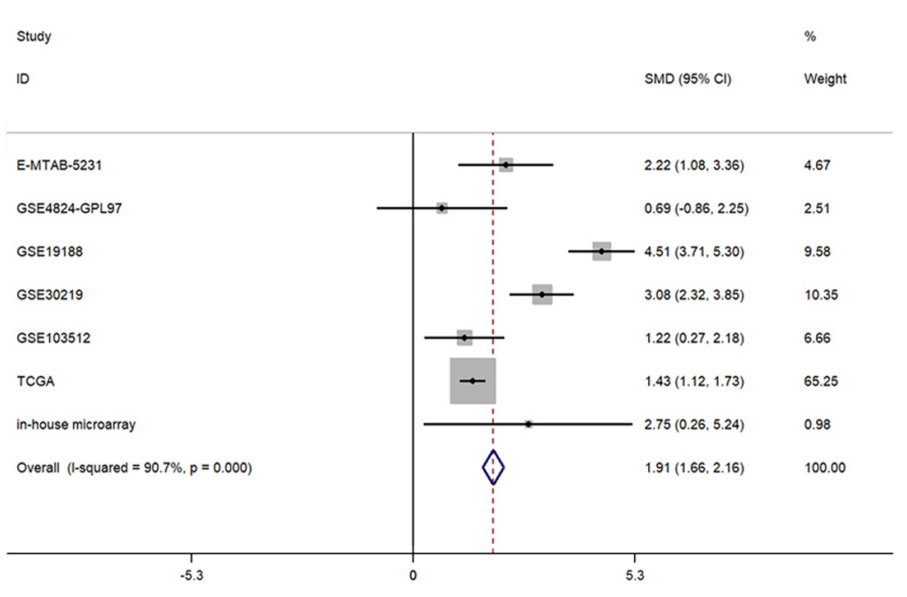

B

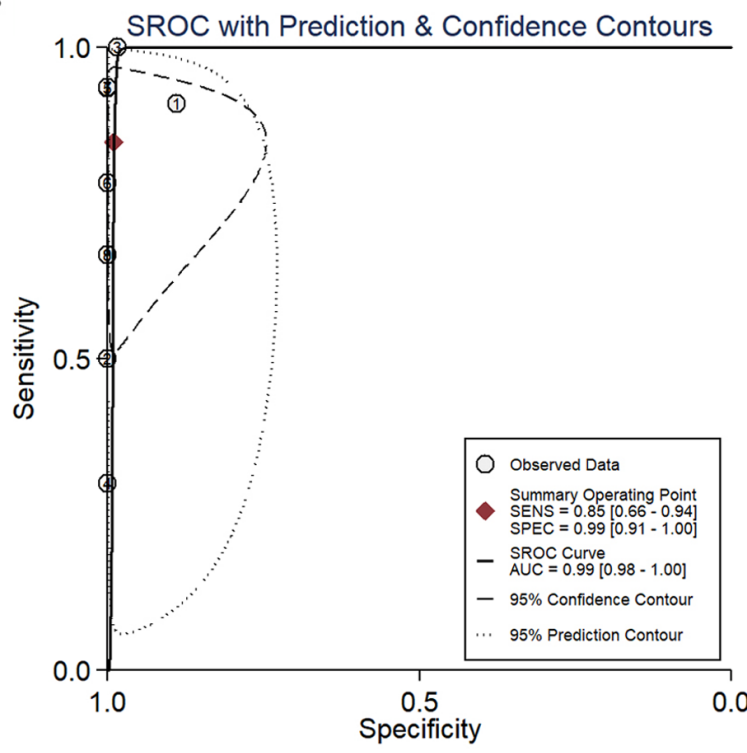

Figure 4. Expression of BBOX1-AS1 and the SROC curve for the included datasets. (A) Forest plot of datasets evaluating BBOX1-AS1 expression between the LUSC and normal control groups (random-effects model). (B) SROC curve for BBOX1-AS1 to discriminate LUSC. SMD, standard mean deviation; SROC, summary receiver operating characteristic; TCGA, The Cancer Genome Atlas; LUSC, lung squamous cell carcinoma; BBOX1-AS1, $\gamma$-butyrobetaine hydroxylase 1 antisense 1; AUC, area under the curve; SENS, sensitivity; SPEC, specificity.

Records excluded due to:

Not human sapniens records;

Not lung squamous cell carcinoma-

related records;

Duplicate records;

Not IncRNA-related records;

Not include cancerous samples and noncancerous specimens;

Could not access the expression value of BBOX1-AS1.

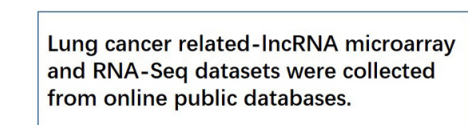

from online public databases.

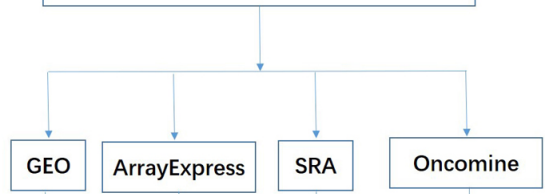

ArrayExpress

Oncomine

Records identified through an initial online databases searching: GEO (35380),

ArrayExpress (502), SRA(0), Oncomine (0).

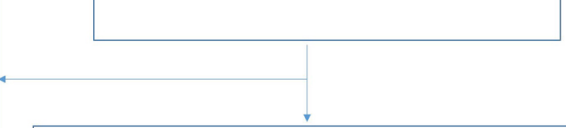

Eligible records for this investigation: GEO (5)

ArrayExpress (1).
BBOX1-AS1 related publications wer searched via online database

PubMed, Web of Science, Science Direct, Google Scholar, Ovid, LILACS, Wiley Online Library, EMBASE,

Cochrane Central Register of Controlled Trials, Chong Qing VIP, Chinese CNKI, Wan Fang and China Biology Medicine disc

No publicaitons identified through initial online databases searching: $n=2$

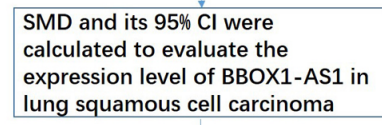

SMD and its $95 \% \mathrm{Cl}$ were

expression level of BBOX1-AS1 in

lung squamous cell carcinoma
SROCs were generated to access the potential utility in distinguishing LUSC and noncancer cases.

Clinical value of BBOX1-AS1 in lung squamous cell carcinoma was evaluated.

Figure 5. Flow chart of screening the included datasets in the SMD and SROC analysis. SMD, standard mean deviation; SROC, summary receiver operating characteristic; GEO Gene Expression Omnibus; lncRNA, long noncoding RNA; RNA-Seq, RNA sequencing; BBOX1-AS1, $\gamma$-butyrobetaine hydroxylase 1 antisense 1; SRA, Sequence Read Archive.

with high BBOX1-AS1 expression was determined via Kaplan-Meier curve analysis ( $\mathrm{P}=0.505$; Fig. 3D).
The RT-qPCR results suggested that BBOX1-AS1 was more highly expressed in LUSC cells (NCI-H1703, NCI-H266, 
A

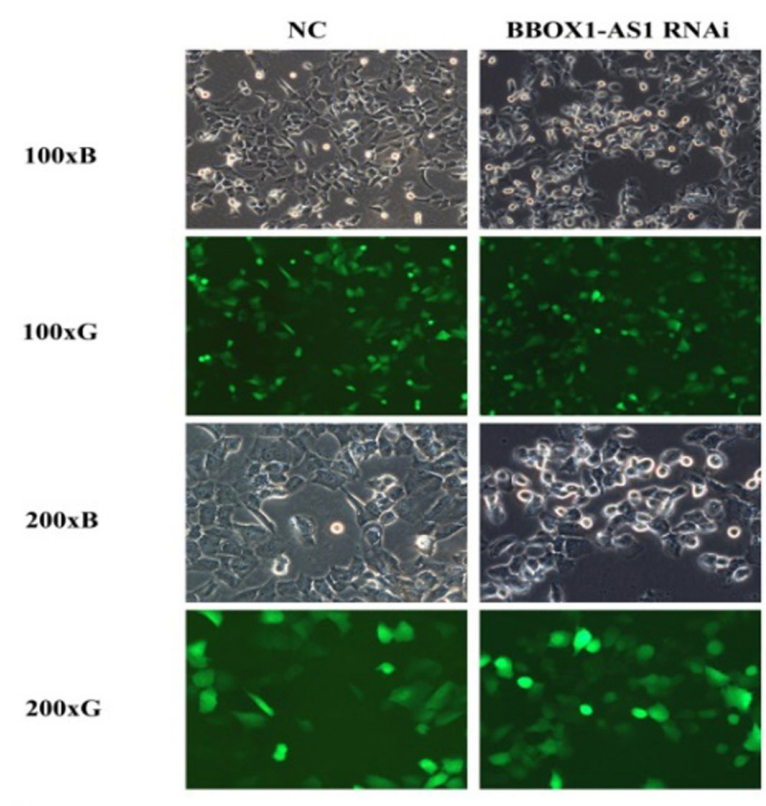

C

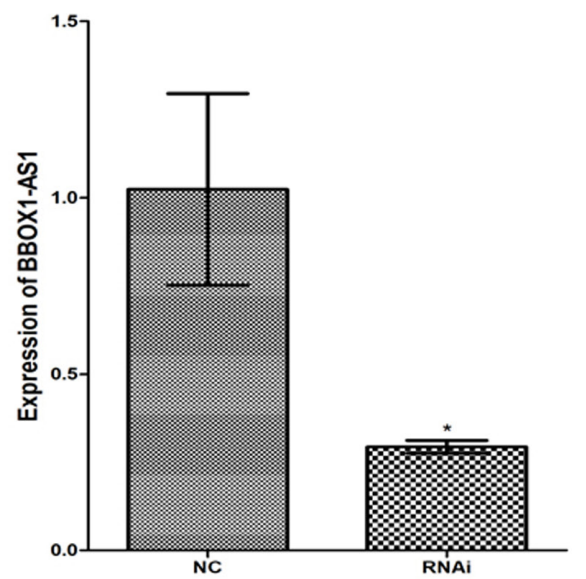

B NCI-H1703

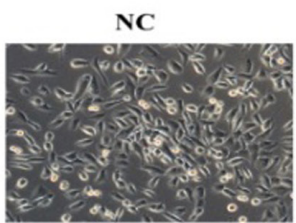

BBOX1-AS1 RNAi
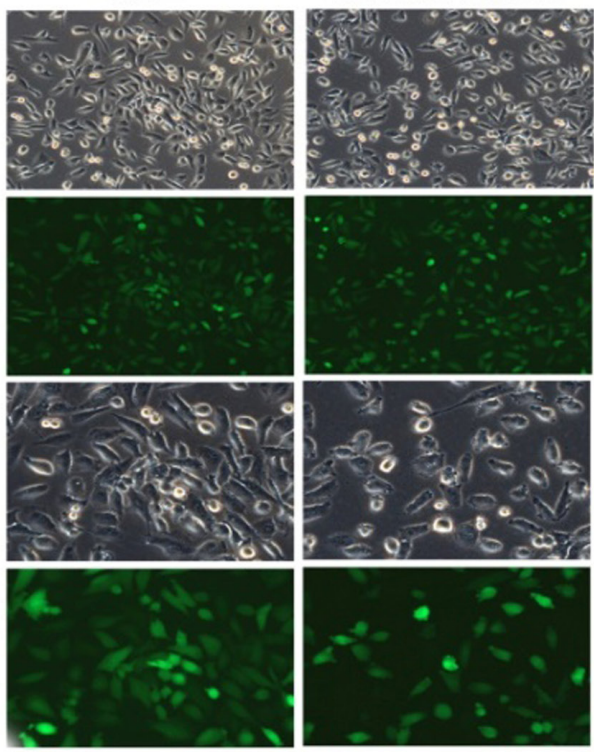

D

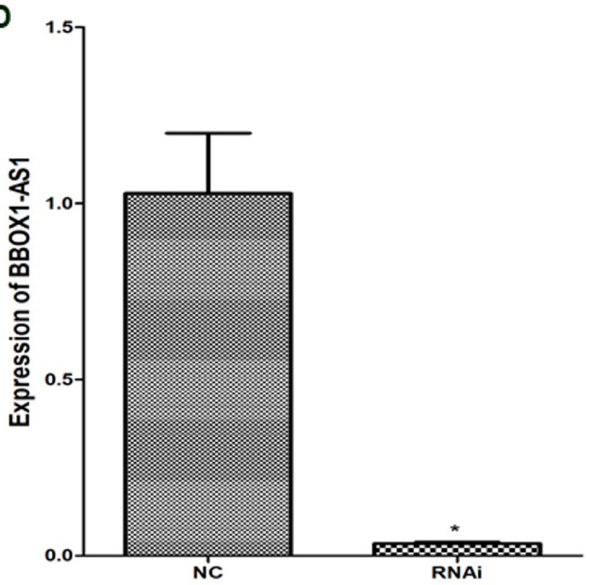

Figure 6. Images of lentivirus-transfected lung squamous cell carcinoma cells and the expression of BBOX1-AS1 in different groups after transfection. (A and B) Images of (A) SK-MES-1 and (B) NCI-H1703 cells transfected with lentivirus after $72 \mathrm{~h}$ (magnification, x100 and x200). B and G indicate transmitted light and fluorescence microscopy images, respectively. (C and D) Differential expression of BBOX1-AS1 in (C) SK-MES-1 and (D) NCI-H1703 cells after transfection determined by reverse transcription-quantitative PCR. "P<0.05. BBOX1-AS1, $\gamma$-butyrobetaine hydroxylase 1 antisense 1; NC, negative control; RNAi, RNA interference.

SK-MES-1) than in BEAS-2B cells, particularly in NCI-H1703 and SK-MES-1 cells (Fig. 3E). NCI-H1703 and SK-MES-1 cells were selected for further research. The original data from six datasets (E-MTAB-5231, GSE4824-GPL97, GSE19188, GSE27489, GSE30219 and GSE103512) from GEO and the ArrayExpress database were used to verify the expression of BBOX1-AS1 in LUSC. Of these, four datasets (E-MTAB-5231, GSE19188, GSE30219 and GSE103512) confirmed high expression of BBOX1-AS1 (Fig. 3F-I).

The results of the SMD analysis (including 641 cases of LUSC and 159 controls) indicated that the combined SMD and 95\% CI reached 1.91 (1.66, 2.16), suggesting significantly higher BBOX1-AS1 expression in the LUSC group than in the control group ( $\mathrm{P}<0.001$; Fig. 4A). Of note, publication bias was detected in the present study $(\mathrm{P}<0.05$; Fig. $\mathrm{S} 1 \mathrm{~A}$ and $\mathrm{B})$. The pooled sensitivity and specificity of BBOX1-AS1 were 0.85 (0.66-0.94) and 0.99 (0.91-1.00), respectively (Fig. S1C). The positive diagnostic likelihood ratio (DLR-positive) and negative diagnostic likelihood ratio (DLR-negative) scores were 83.24 (9.10-761.45) and $0.15(0.06-0.38)$, respectively (Fig. S1D). LR $<0.1$ or $>10.0$ was indicative of high accuracy. The diagnostic score and odds ratio were 6.30 (3.82-8.77) and 542.07 (45.73-6425.35), respectively (Fig. S1E). The AUC of the SROC was 0.99 (0.98-1.00; Fig. 4B), which indicated a high potential utility of BBOX-AS1 for distinguishing between LUSC and noncancer cases. Publication bias occurred in the SROC analysis $(\mathrm{P}<0.05$; Fig. S1B). A flow chart of the SMD and SROC analyses is provided in Fig. 5.

Effect of BBOX1-AS1 on the proliferation and migration of LUSC cells in vitro. The transfection efficiency of BBOX1-AS1 RNAi plasmid was observed using a light microscope and fluorescence microscope. The transfection efficiency in the BBOX1-AS1 RNAi group in LUSC cell lines was $>80 \%$ and the knockdown efficiency of BBOX1-AS1 was $>70 \%$. Images of cells after transfection with BBOX1-AS1 RNAi and control lentivirus are provided 

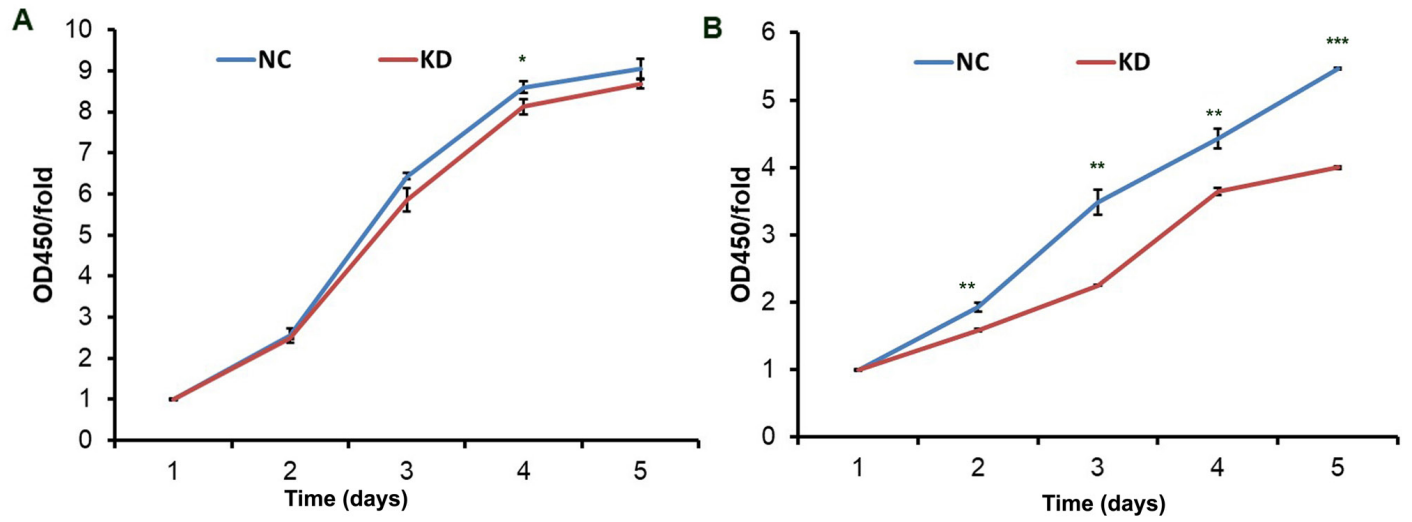

Figure 7. Detection of the effect of $\gamma$-butyrobetaine hydroxylase 1 antisense 1 on the proliferation ability of lung squamous cell carcinoma cells by Cell Counting Kit-8 assay. (A) NCI-H1703 cells; (B) SK-MES-1 cells. " $\mathrm{P}<0.05,{ }^{* *} \mathrm{P}<0.01,{ }^{* * * *} \mathrm{P}<0.001$ for KD vs. NC at the same time-point. OD450, optical density at $450 \mathrm{~nm}$; NC, negative control; KD, knockdown.

A

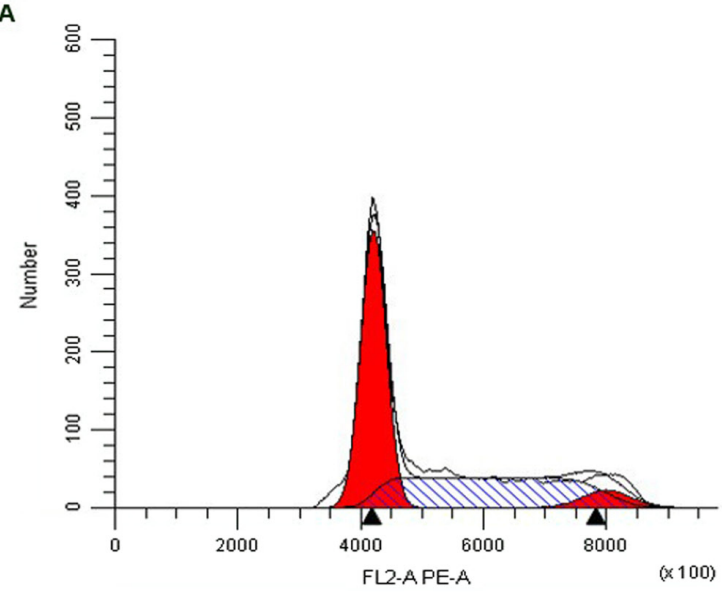

B

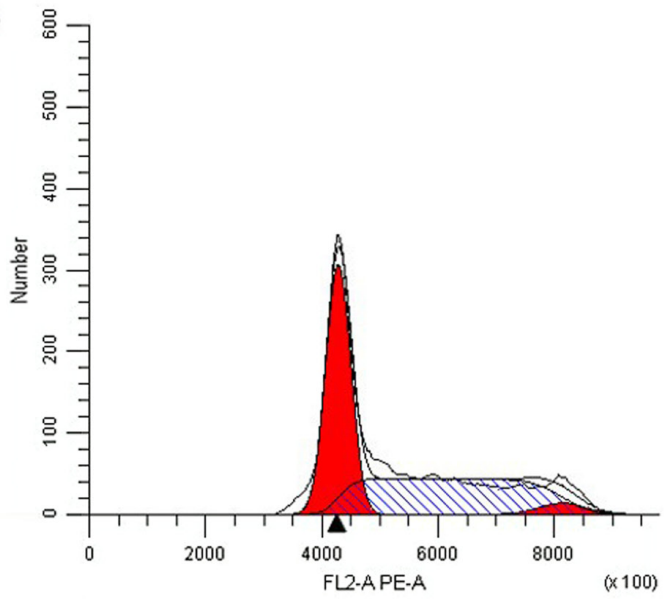

C

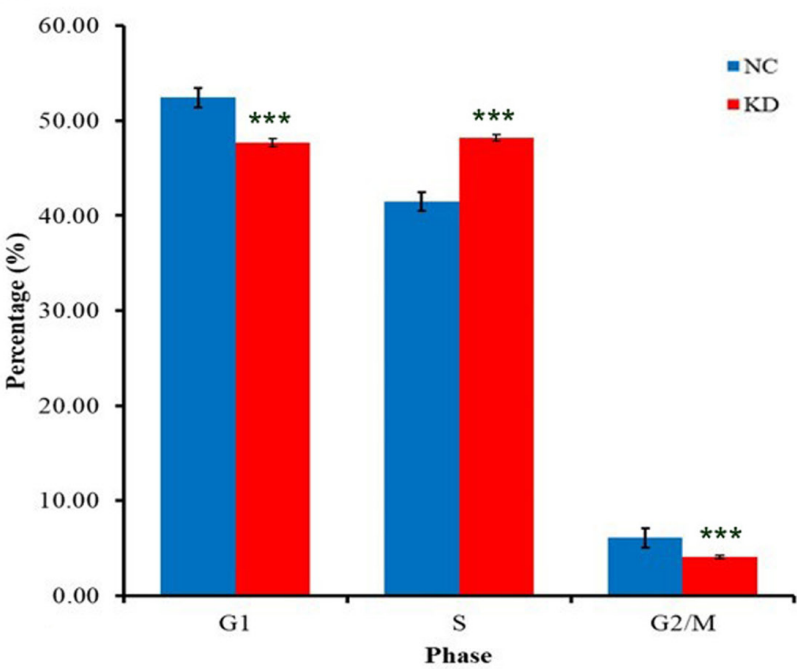

Figure 8. Cell cycle distribution of NCI-H1703 cells after knockdown of $\gamma$-butyrobetaine hydroxylase 1 antisense 1 expression. (A and B) Flow cytometry images for the (A) NC group and (B) KD group; (C) distribution of NCI-H1703 cells in G1, S and G2/M phase. ${ }^{* * * *} \mathrm{P}<0.001$ vs. NC. NC, negative control; $\mathrm{KD}$, knockdown.

in Fig. 6A and B. RT-qPCR indicated that BBOX1-AS1 expression was significantly lower in the BBOX1-AS1 RNAi groups than in the control groups in both NCI-H1703 and SK-MES-1 cells $(\mathrm{P}<0.05$; Fig. 6C and $\mathrm{D})$.
According to the CCK-8 assay, proliferation was inhibited in the KD group in two cell lines (NCI-H1703 and SK-MES-1) compared to the NC groups (Fig. 7). In NCI-H1703 and SK-MES-1 cells, no obvious difference was observed between 
Table II. Top enrichment GO terms in the categories BP, CC and MF by the co-expressed genes of $\gamma$-butyrobetaine hydroxylase 1 antisense 1 .

\begin{tabular}{llccc}
\hline GO ID & \multicolumn{1}{c}{ Term } & Ontology & Count & P-value \\
\hline GO:0034621 & Cellular macromolecular complex subunit organization & BP & 10 & 0.004493 \\
GO:0034622 & Cellular macromolecular complex assembly & BP & 9 & 0.007375 \\
GO:0042476 & Odontogenesis & BP & 4 & 0.012155 \\
GO:0048730 & Epidermis morphogenesis & BP & 3 & 0.020492 \\
GO:0007155 & Cell adhesion & BP & 13 & 0.021424 \\
GO:0022610 & Biological adhesion & BP & 13 & 0.021636 \\
GO:0048729 & Tissue morphogenesis & BP & 6 & 0.022049 \\
GO:0007229 & Integrin-mediated signaling pathway & BP & 4 & 0.024231 \\
GO:0048732 & Gland development & BP & 5 & 0.032087 \\
GO:0007398 & Ectoderm development & BP & 6 & 0.032126 \\
GO:0030057 & Desmosome & CC & 5 & $1.88 \mathrm{E}-05$ \\
GO:0070161 & Anchoring junction & CC & 8 & $5.32 \mathrm{E}-04$ \\
GO:0005911 & Cell-cell junction & CC & 7 & 0.004754 \\
GO:0043296 & Apical junction complex & CC & 5 & 0.008914 \\
GO:0016327 & Apicolateral plasma membrane & CC & 5 & 0.009878 \\
GO:0044427 & Chromosomal part & CC & 4 & 0.0141 \\
GO:0031965 & Nuclear membrane & CC & 9 & 0.022122 \\
GO:0005694 & Chromosome & CC & 7 & 0.035488 \\
GO:0019866 & Organelle inner membrane & CC & 9 & 0.05378 \\
GO:0030054 & Cell junction & MF & 5 & 0.062978 \\
GO:0032403 & Protein complex binding & MF & 3 & 0.094009 \\
GO:0005178 & Integrin binding & & & 54724 \\
\hline
\end{tabular}

GO, Gene Ontology; BP, biological process; CC, cellular component; MF, molecular function.

A

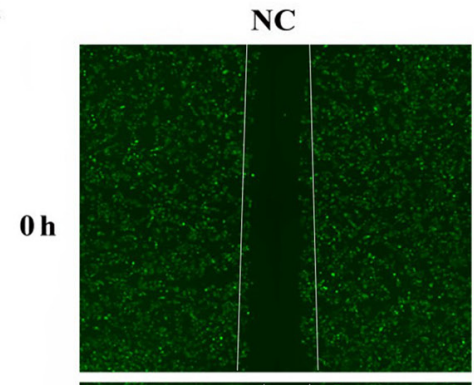

BBOX1-AS1 KD
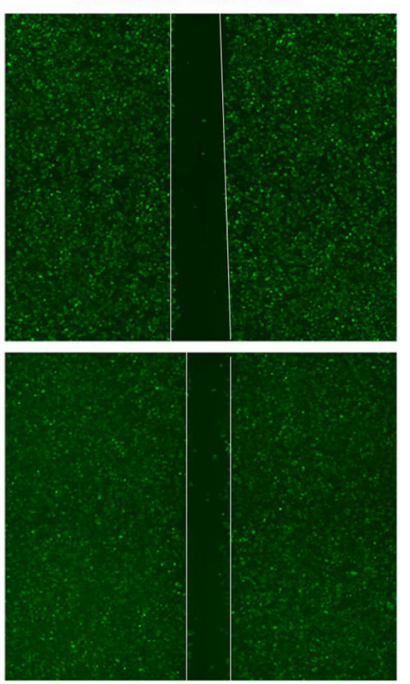

B

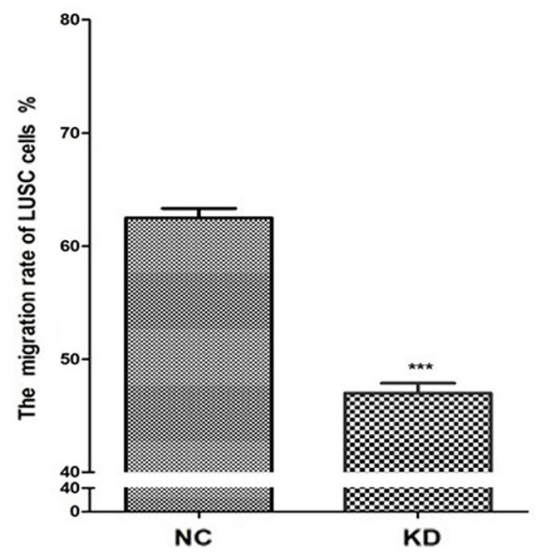

Figure 9. Migration ability of SK-MES-1 cells after knockdown of BBOX1-AS1 expression. (A) Scratch-assay images for the NC group and KD group at 0 and $24 \mathrm{~h}$ (magnification, $\mathrm{x} 40$ ). (B) Migration ability of cells in different groups. ${ }^{* * * *} \mathrm{P}<0.001$ vs. NC. NC, negative control; KD, knockdown; BBOX1-AS1, $\gamma$-butyrobetaine hydroxylase 1 antisense 1 .

the KD group and the NC group on the first day. The proliferation of SK-MES-1 cells in the KD group was markedly reduced on days 2-5 ( $\mathrm{P}<0.05)$ and most significantly reduced on day five.
There was no clear difference observed in NCI-H1703 cells on the second and third days, whereas a significant decrease was determined on day four $(\mathrm{P}<0.05)$. Based on these results, it 

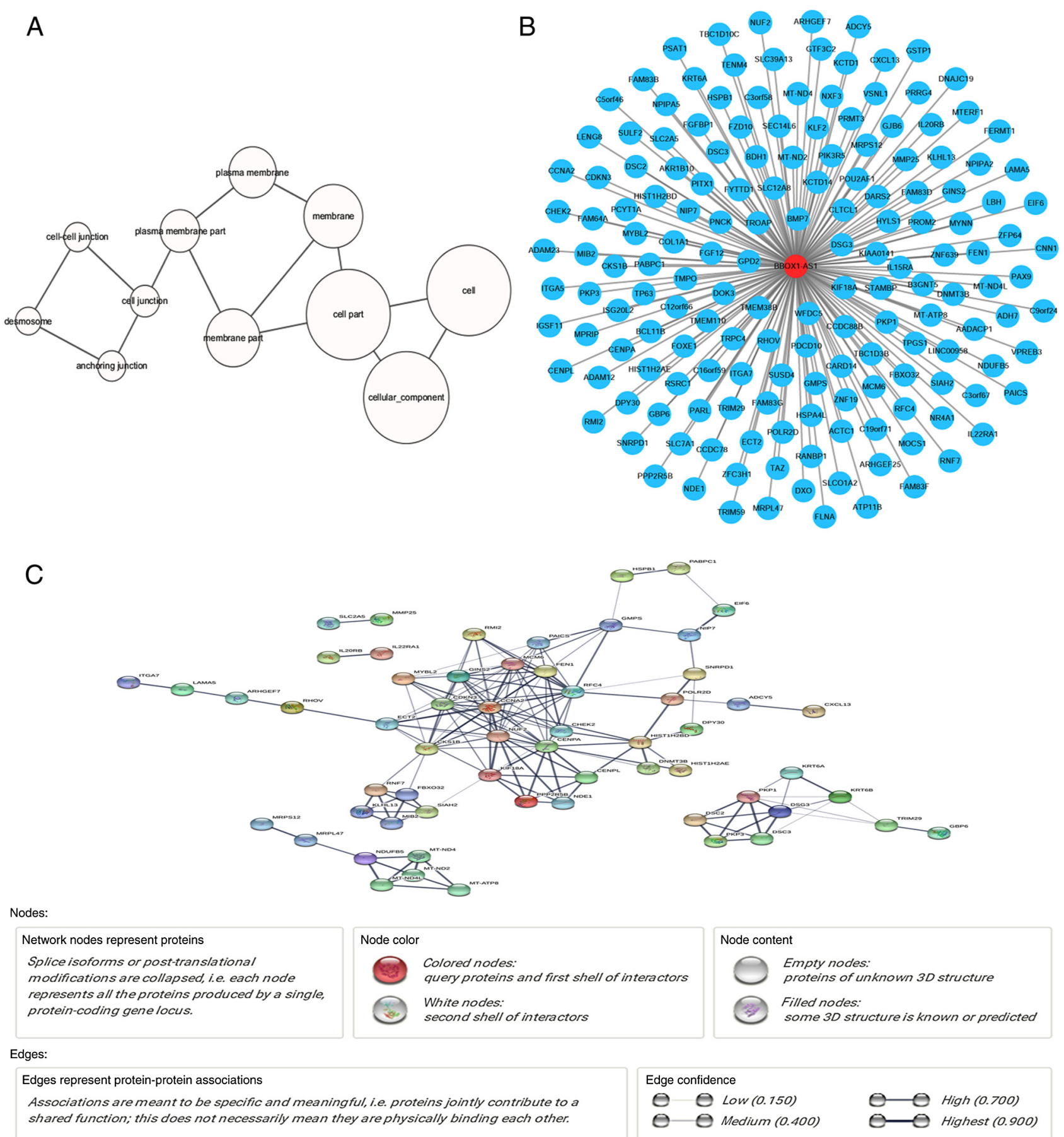

Figure 10. GO analysis and network analysis of the genes coexpressed with BBOX1-AS1. (A) GO analysis of the genes coexpressed with BBOX1-AS1. (B) Network analysis between BBOX1-AS1 and the coexpressed genes. The relationships between BBOX1-AS1 and the coexpressed genes were easily observed from this network. (C) The PPI network was constructed via STRING online and 253 PPI pairs were chosen for further analysis. GO, gene ontology; BBOX1-AS1, $\gamma$-butyrobetaine hydroxylase 1 antisense 1; PPI, protein-protein interaction.

may be concluded that silencing of BBOX1-AS1 inhibited the proliferation of LUSC cells.

In NCI-H1703 cells, the results of the cell cycle analysis revealed that BBOX1-AS1 RNAi caused cell cycle arrest in $\mathrm{S}$ phase ( $\mathrm{P}<0.05$; Fig. 8). Thus, BBOX1-AS1 RNAi may inhibit the proliferation of LUSC cells partly because silencing of BBOX1-AS1 arrested cell cycle progression.

Furthermore, the results of the Celigo scratch assay suggested that migration was inhibited after knockdown of BBOX1-AS1 in SK-MES-1 cells ( $\mathrm{P}<0.01$; Fig. 9A and B).
Potential pathways associated with BBOX1-AS1. The in-house microarray, TCGA and GEO datasets were used to explore the genes co-expressed with BBOX1-AS1. A total of 167 genes were selected for downstream analysis. The possible GO and KEGG pathways were examined based on these 167 genes. The GO and KEGG analyses suggested that these genes may participate in various important biological processes, including cell-cell junctions, protein complex binding, tissue morphogenesis, focal adhesion, ECM-receptor interaction and DNA replication (Fig. 10A; Tables II and III). 
Table III. Enriched KEGG pathway terms by the co-expressed genes of $\gamma$-butyrobetaine hydroxylase 1 antisense 1.

\begin{tabular}{llcll}
\hline KEGG ID & \multicolumn{1}{c}{ KEGG term } & Count & P-value & \multicolumn{1}{c}{ Gene symbols } \\
\hline hsa04510 & Focal adhesion & 6 & 0.03381 & ITGA5, LAMA5, ITGA7, PIK3R5, COL1A1, FLNA \\
hsa04512 & ECM-receptor interaction & 4 & 0.039674 & ITGA5, LAMA5, ITGA7, COL1A1 \\
hsa03030 & DNA replication & 3 & 0.041514 & RFC4, FEN1, MCM6 \\
hsa05414 & Dilated cardiomyopathy & 4 & 0.049758 & ACTC1, ITGA5, ADCY5, ITGA7 \\
\hline
\end{tabular}

KEGG, Kyoto Encyclopedia of Genes and Genomes.
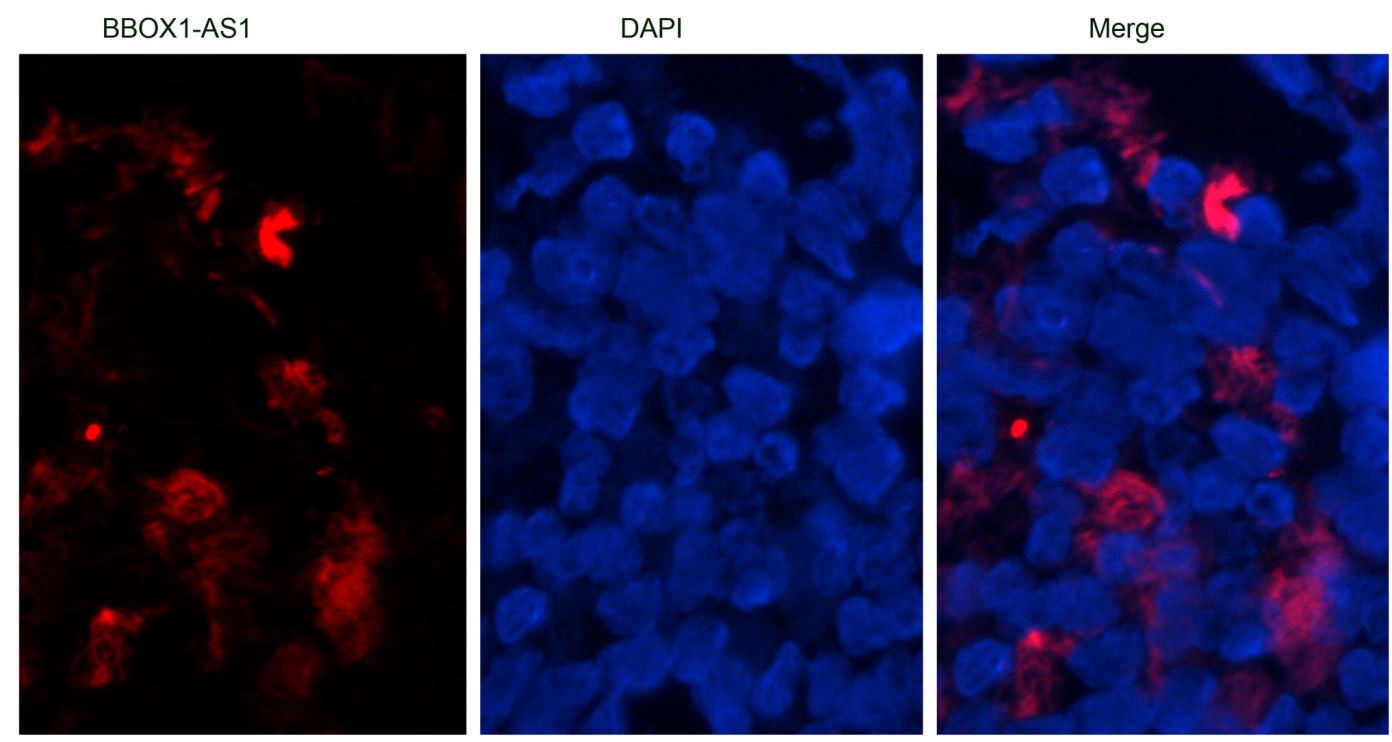

Figure 11. Cellular localization of BBOX1-AS1 in lung squamous cell carcinoma cells as detected via FISH. FISH was performed to observe the cellular location of BBOX1-AS1 (red) in cells (magnification, x400). FISH, fluorescence in situ hybridization; BBOX1-AS1, $\gamma$-butyrobetaine hydroxylase 1 antisense 1.

A gene network of the co-expressed genes of BBOX1-AS1 was also constructed (Fig. 10B), from which it was possible to detect relationships between BBOX1-AS1 and these co-expressed genes.

The PPI network was constructed using STRING online and 253 PPI pairs with a combined score $>0.4$ were selected (Fig. 10C). The interactions between BBOX1-AS1 and coexpressed genes were obtained through the nodes and strings in the network. Centromere protein A (CENPA) had the highest degree of connectivity (degree $=15$ ) according to the PPI network, which indicates that CENPA may be the key gene coexpressed with BBOX1-AS1 in LUSC. In addition, CENPA was downregulated in LUSC based on the TCGA and GEPIA datasets (Fig. S2A and B). Furthermore, the correlation between BBOX1-AS1 and CENPA was determined with the TCGA and GEPIA datasets, indicating that BBOX1-AS1 expression was positively correlated with CENPA in LUSC $(\mathrm{R}=0.1512$; Fig. S2C and D).

Cellular localization of BBOXI-AS1. FISH analysis was performed to observe the cellular location of BBOX1-AS1 in LUSC cells. The majority of BBOX1-AS1 (indicated in red) was located in the cytoplasm (magnification, $\mathrm{x} 400$ ), indicating that BBOX1-AS1 is able to act as a competing endogenous (ce) RNA in LUSC (Fig. 11).

\section{Discussion}

Previous studies have revealed the pivotal role of BBOX1-AS1 in NSCLC, as well as colorectal, cervical and gastric cancers (17-20). Liu et al (17) indicated that BBOX1-AS1 was overexpressed in colorectal cancer cell lines and that BBOX1-AS1 knockdown enhanced cell proliferation, invasion and migration while decreasing apoptosis. In cervical cancer, $\mathrm{Xu}$ et al (19) determined that BBOX1-AS1 was upregulated. Increased expression of BBOX1-AS1 had stimulating effects on cervical cancer cell growth and migration. Yang et al (18) determined that BBOX1-AS1 was significantly upregulated in gastric cancer tissues and cells and that overexpression of BBOX1-AS1 promoted gastric cancer cell proliferation and inhibited gastric cancer cell apoptosis. Shi et al (20) revealed that KLF5-induced BBOX1-AS1 expression exerted a tumor-promoting role in NSCLC by sponging microRNA (miRNA/miR)-27a-5p. Several obvious differences were observed between the present study and that by Shi et al (20). First, the present study used an in-house microarray analysis to detect differentially expressed IncRNAs between LUSC and normal lung tissues. BBOX1-AS1 was the only overlapping upregulated lncRNA based on the datasets from the TCGA, GEO, ArrayExpress and the in-house microarray. Next, BBOX1-AS1 was indicated to act as a competing 'sponge' of miRNA, but this requires BBOX1-AS1 
to be located in the cytoplasm. FISH analysis was performed in the present study to determine the location of BBOX1-AS1 in the nucleus or cytoplasm of LUSC tissues. Furthermore, SMD and SROC analyses were included in the present study. SMD analysis confirmed upregulated expression of BBOX1-AS1 in LUSC. SROC analysis indicated the high potential utility of BBOX-AS1 in distinguishing between LUSC and noncancer cases.

In the present study, RT-qPCR and in vitro verification were used to further determine the effect of BBOX1-AS1 in LUSC. LUSC cell proliferation and migration were reduced following BBOX1-AS1 RNAi. These discoveries confirmed the oncogenic role of BBOX1-AS1 in LUSC. NCI-H1703, NCI-H226 and SK-MES-1 cells are three well-known human LUSC cell lines. According to the RT-qPCR analysis, BBOX1-AS1 expression was higher in NCI-H1703 and SK-MES-1 cells than in NCI-H226 cells. Therefore, the NCI-H1703 and SK-MES-1 cell lines were selected for further transfection with BBOX1-AS1 RNAi, and it was indicated that the tumor cell clones were heterogeneous with respect to both morphology and function. Lung cancer is highly heterogeneous in cell origin, histology, clinical features, molecular biology and other aspects, resulting in a lack of effective and specific therapy strategies (33). Future therapies will undoubtedly focus on precision medicine and personalized treatments as the study of nosogenesis continues.

In the present study, it was discovered that high BBOX1-AS1 expression was associated with lymphatic metastasis, which is an important clinical finding in LUSC. Furthermore, the high AUC value indicated that BBOX1-AS1 expression is able to discriminate between LUSC and noncancerous tissues. First, lncRNA high-throughput chips and original RNA-seq data were collected from multiple databases to calculate the SMD and SROC curve of BBOX1-AS1 for LUSC. The combined values of sensitivity (0.85) and specificity (0.99) demonstrated the high accuracy of BBOX1-AS1 for the detection of LUSC. The present results verified that the SROC curve was located near the upper left corner. PLR and NLR were determined to predict the diagnostic accuracy of BBOX1-AS1. $\mathrm{LR}<0.1$ or $>10.0$ was indicative of high accuracy. A PLR value of 83.24 suggested that patients with LUSC had an 83.24-fold higher chance of expressing high levels of BBOX1-AS1. In addition, the NLR (0.15) suggested that if the BBOX1-AS1 result was negative, there was an $\sim 15 \%$ chance that the patient had LUSC. SMD analysis confirmed higher expression of BBOX1-AS1 in LUSC, in accordance with the results of the RT-qPCR and in-house microarray analyses.

The GO and KEGG analyses indicated that the target genes may have various biological roles. Previous studies suggested that these enriched terms may have essential functions in various cancer types, including cervical, lung and liver cancer $(34,35)$. These enriched functions may be associated with tumor occurrence, and the progression and development of lung cancer $(34,36)$. CENPA had the highest degree of involvement based on the PPI network. CENPA was reported to be significantly involved in the prognosis and predicted outcome of targeted therapy of LUSC $(37,38)$. It was hypothesized that CENPA is the key gene downstream of BBOX1-AS1 in LUSC. BBOX1-AS1 may affect the progression and development of LUSC by regulating various biological functions. However, the precise molecular mechanisms should be determined in future functional experiments.
lncRNAs may be located in the cytoplasm and function as ceRNAs in cancer $(39,40)$. Kong et al (39) reported that lncRNA-CDC6 may act as a ceRNA to target CDC6 by sponging miR-215 to promote the progression of breast cancer. Tang and Yang (40) indicated that lncRNA SNHG14 may function as a ceRNA in hepatocellular carcinoma and the SNHG14/miR-656-3p/SIRT5 axis was able to aggravate the invasion and migration of hepatocellular carcinoma. The results of the FISH assay suggested that BBOX1-AS1 was located in the cytoplasm. Thus, BBOX1-AS1 may act as a ceRNA to affect the biological functions of LUSC. BBOX1-AS1/miR-361-3p/AEG-1 may have significant roles in the proliferation, invasion and metastasis of LUSC based on the literature and bioinformatic prediction software, but functional experiments should be performed for verification $(17,19,41)$.

There were certain limitations to the SMD and SROC analyses. Publication bias was detected and heterogeneity (high I-square values) was unavoidable due to the nonsignificant results in the two GEO datasets. Furthermore, the use of blinding was inconsistent among the five different data sources (GEO, ArrayExpress, TCGA, RT-qPCR and in-house microarray), which contributed to the heterogeneity. To the best of our knowledge, the present study was the first to explore the relationship between BBOX1-AS1 expression and the biological activity of LUSC in vitro. It was determined that BBOX1-AS1 was able to promote the proliferation and migration of LUSC cells. Additional functional experiments should be performed to verify the potential molecular mechanism of BBOX1-AS1 in LUSC.

In conclusion, the present study indicated that BBOX1-AS1 may have an oncogenic effect in LUSC by regulating various biological functions. However, additional functional experiments should be designed to verify the exact mechanism.

\section{Acknowledgements}

Not applicable.

\section{Funding}

The present study was supported by the Natural Science Foundation of Shandong, China (grant no. ZR202102240355).

\section{Availability of data and materials}

The datasets used and/or analyzed during the present study are available from the corresponding author upon reasonable request.

\section{Authors' contributions}

YZ and XW made a significant contribution to the study design, execution and acquisition of data. XKC, YYZ and RQH contributed to data analysis and interpretation. GC and YJQ analyzed the experimental data and wrote and revised the manuscript. YZ and GC confirm the authenticity of the raw data. All authors read and approved the final version of the manuscript.

\section{Ethics approval and consent to participate}

All methods used adhered to the relevant guidelines and were approved by the Ethical Committee of the First Affiliated 
Hospital of Guangxi Medical University (Nanning, China). Consent forms for the use of tissues were signed by the doctors and patients involved in the study.

\section{Patient consent for publication}

Not applicable.

\section{Competing interests}

The authors declare that they have no competing interests.

\section{References}

1. Zhao Y, Wang XX, Wu W, Long H, Huang J, Wang Z, Li T, Tang S, Zhu B and Chen D: EZH2 regulates PD-L1 expression via HIF- $1 \alpha$ in non-small cell lung cancer cells. Biochem Biophys Res Commun 517: 201-209, 2019.

2. Laurans M, Botticella A, Moukasse Y,Lévy A and Le Péchoux C: Lung cancer and elective nodal irradiation: A solved issue? Cancer Radiother 23: 701-707, 2019 (In French).

3. Buentzel J, Heinz J, Bleckmann A, Bauer C, Röver C, Bohnenberger H, Saha S, Hinterthaner M, Baraki H, Kutschka I, et al: Sarcopenia as Prognostic Factor in Lung Cancer Patients: A Systematic Review and Meta-analysis. Anticancer Res 39: 4603-4612, 2019.

4. Huang N,Lin W, Shi X and Tao T: STK24 expression is modulated by DNA copy number/methylation in lung adenocarcinoma and predicts poor survival. Future Oncol 14: 2253-2263, 2018.

5. Hoang LT, Domingo-Sabugo C, Starren ES, Willis-Owen SAG, Morris-Rosendahl DJ, Nicholson AG, Cookson WOCM and Moffatt MF: Metabolomic, transcriptomic and genetic integrative analysis reveals important roles of adenosine diphosphate in haemostasis and platelet activation in non-small-cell lung cancer. Mol Oncol 13: 2406-2421, 2019.

6. Fu L, Wang H, Wei D, Wang B, Zhang C, Zhu T, Ma Z, Li Z, Wu Y and Yu G: The value of CEP55 gene as a diagnostic biomarker and independent prognostic factor in LUAD and LUSC. PLoS One 15: e233283, 2020

7. Li K, Tian Y, Yuan Y, Fan X, Yang M, He Z and Yang D: Insights into the Functions of lncRNAs in Drosophila. Int J Mol Sci 20 20, 2019 .

8. Murillo-Maldonado JM and Riesgo-Escovar JR: The various and shared roles of lncRNAs during development. Dev Dyn 248: 1059-1069, 2019.

9. Zhu J, Yu W, Wang Y, Xia K, Huang Y, Xu A, Chen Q, Liu B, Tao H, Li F, et al: IncRNAs: Function and mechanism in cartilage development, degeneration, and regeneration. Stem Cell Res Ther 10: 344, 2019.

10. Wang S, Jin J, Xu Z and Zuo B: Functions and Regulatory Mechanisms of lncRNAs in Skeletal Myogenesis, Muscle Disease and Meat Production. Cells 8: 8, 2019.

11. Lim LJ, Wong SYS, Huang F, Lim S, Chong SS, Ooi LL, Kon OL and Lee CG: Roles and Regulation of Long Noncoding RNAs in Hepatocellular Carcinoma. Cancer Res 79: 5131-5139, 2019.

12. Chi Y, Wang D, Wang J, Yu W and Yang J: Long Non-Coding RNA in the Pathogenesis of Cancers. Cells 8: 8, 2019.

13. Yang J, Qiu Q, Qian X, Yi J, Jiao Y, Yu M, Li X, Li J, Mi C, Zhang $\mathrm{J}$, et al: Long noncoding RNA LCAT1 functions as a ceRNA to regulate RAC1 function by sponging miR-4715-5p in lung cancer. Mol Cancer 18: 171, 2019.

14. Zhao Y, Feng C, Li Y, Ma Y and Cai R: IncRNA H19 promotes lung cancer proliferation and metastasis by inhibiting miR-200a function. Mol Cell Biochem 460: 1-8, 2019.

15. Huang N, Guo W, Ren K, Li W, Jiang Y, Sun J, Dai W and Zhao W: lncRNA AFAP1-AS1 Supresses miR-139-5p and Promotes Cell Proliferation and Chemotherapy Resistance of Non-small Cell Lung Cancer by Competitively Upregulating RRM2. Front Oncol 9: 1103, 2019.

16. Jin D, Guo J, Wu Y, Du J, Yang L, Wang X, Di W, Hu B, An J, Kong L, et al: m6A mRNA methylation initiated by METTL3 directly promotes YAP translation and increases YAP activity by regulating the MALAT1-miR-1914-3p-YAP axis to induce NSCLC drug resistance and metastasis. J Hematol Oncol 12 $135,2019$.
17. Liu J, Zhu J, Xiao Z, Wang X and Luo J: BBOX1-AS1 contributes to colorectal cancer progression by sponging hsa-miR-361-3p and targeting SH2B1. FEBS Open Bio 2211-5463.12802, 2020.

18. Yang Y, Yu Q, Li B, Guan R, Huang C and Yang X: BBOX1-AS1 Accelerates Gastric Cancer Proliferation by Sponging miR-3940-3p to Upregulate BIRC5 Expression. Dig Dis Sci 66: 1054-1062, 2021.

19. Xu J, Yang B, Wang L, Zhu Y, Zhu X, Xia Z, Zhao Z and Xu L: lncRNA BBOX1-AS1 upregulates HOXC6 expression through miR-361-3p and HuR to drive cervical cancer progression. Cell Prolif 53: e12823, 2020.

20. Shi J, Yang C, An J, Hao D, Liu C, Liu J, Sun J and Jiang J: KLF5-induced BBOX1-AS1 contributes to cell malignant phenotypes in non-small cell lung cancer via sponging miR-27a-5p to up-regulate MELK and activate FAK signaling pathway. J Exp Clin Cancer Res 40: 148, 2021.

21. Zhang Y, He RQ, Dang YW, Zhang XL, Wang X, Huang SN, Huang WT, Jiang MT, Gan XN, Xie Y, et al: Comprehensive analysis of the long noncoding RNA HOXA11-AS gene interaction regulatory network in NSCLC cells. Cancer Cell Int 16: 89, 2016.

22. Linehan WM and Ricketts CJ: The Cancer Genome Atlas of renal cell carcinoma: Findings and clinical implications. Nat Rev Urol 16: 539-552, 2019.

23. Xia L, Zhang W and Gao L: Clinical and prognostic effects of CDKN2A, CDKN2B and CDH13 promoter methylation in ovarian cancer: A study using meta-analysis and TCGA data. Biomarkers 24: 700-711, 2019.

24. Cai KT,Liu AG, Wang ZF, Jiang HW,Zeng JJ,He RQ, Ma J, Chen G and Zhong JC: Expression and potential molecular mechanisms of miR $2045 p$ in breast cancer, based on bioinformatics and a meta analysis of 2,306 cases. Mol Med Rep 19: 1168-1184, 2019.

25. Gan BL, Zhang LJ, Gao L, Ma FC, He RQ, Chen G, Ma J, Zhong JC and Hu XH: Downregulation of miR 2245 in prostate cancer and its relevant molecular mechanism via TCGA, GEO database and in silico analyses. Oncol Rep 40: 3171-3188, 2018.

26. Rao X, Huang X, Zhou Z and Lin X: An improvement of the $2^{\wedge}(-$ delta delta CT) method for quantitative real-time polymerase chain reaction data analysis. Biostat Bioinforma Biomath 3: 71-85, 2013.

27. Zhang Y, Chen WJ, Gan TQ, Zhang XL, Xie ZC, Ye ZH, Deng Y, Wang ZF, Cai KT, Li SK, et al: Clinical Significance and Effect of lncRNA HOXA11-AS in NSCLC: A Study Based on Bioinformatics, In Vitro and In Vivo Verification. Sci Rep 7: 5567, 2017.

28. Zhang Y, Li ZY, Hou XX, Wang X, Luo YH, Ying YP and Chen G: Clinical significance and effect of AEG-1 on the proliferation, invasion, and migration of NSCLC: A study based on immunohistochemistry, TCGA, bioinformatics, in vitro and in vivo verification. Oncotarget 8: 16531-16552, 2017.

29. Cheng Q, Huang C, Cao H, Lin J, Gong X, Li J, Chen Y, Tian Z, Fang Z and Huang J: A Novel Prognostic Signature of Transcription Factors for the Prediction in Patients With GBM. Front Genet 10: 906, 2019.

30. Ding J and Zhang Y: Analysis of key GO terms and KEGG pathways associated with carcinogenic chemicals. Comb Chem High Throughput Screen: Dec 18, 2017 (Epub ahead of print). doi: $10.2174 / 1386207321666171218120133$.

31. Zhou Z, Li Y, Hao H, Wang Y, Zhou Z, Wang Z and Chu X: Screening Hub Genes as Prognostic Biomarkers of Hepatocellular Carcinoma by Bioinformatics Analysis. Cell Transplant 28 (Suppl 1): 76S-86S, 2019.

32. Liang Y, Zhang C, Ma MH and Dai DQ: Identification and prediction of novel non-coding and coding RNA-associated competing endogenous RNA networks in colorectal cancer. World J Gastroenterol 24: 5259-5270, 2018

33. de Sousa VML and Carvalho L: Heterogeneity in Lung Cancer. Pathobiology 85: 96-107, 2018.

34. Aboubakar Nana F, Vanderputten M and Ocak S: Role of Focal Adhesion Kinase in Small-Cell Lung Cancer and Its Potential as a Therapeutic Target. Cancers (Basel) 11: 11, 2019.

35. Mughal MJ, Mahadevappa R and Kwok HF: DNA replication licensing proteins: Saints and sinners in cancer. Semin Cancer Biol 58: 11-21, 2019.

36. Yenerall P, Das AK, Wang S, Kollipara RK, Li LS, Villalobos P, Flaming J, Lin YF, Huffman K, Timmons BC, et al: RUVBL1/RUVBL2 ATPase Activity Drives PAQosome Maturation, DNA Replication and Radioresistance in Lung Cancer. Cell Chem Biol 27: 105-121.e14, 2020.

37. Jiang L, Zhao L, Bi J, Guan Q, Qi A, Wei Q, He M, Wei M and Zhao L: Glycolysis gene expression profilings screen for prognostic risk signature of hepatocellular carcinoma. Aging (Albany NY) 11: 10861-10882, 2019. 
38. Qi L, Gao C, Feng F, Zhang T, Yao Y, Wang X, Liu C, Li J, $\mathrm{Li} \mathrm{J}$ and Sun C: MicroRNAs associated with lung squamous cell carcinoma: New prognostic biomarkers and therapeutic targets. J Cell Biochem 120: 18956-18966, 2019.

39. Kong X, Duan Y, Sang Y, Li Y, Zhang H, Liang Y, Liu Y, Zhang $\mathrm{N}$ and Yang Q: IncRNA-CDC6 promotes breast cancer progression and function as ceRNA to target CDC6 by sponging microRNA-215. J Cell Physiol 234: 9105-9117, 2019.

40. Tang SJ and Yang JB: IncRNA SNHG14 aggravates invasion and migration as ceRNA via regulating miR-656-3p/SIRT5 pathway in hepatocellular carcinoma. Mol Cell Biochem 473: 143-153, 2020.
41. Yao H, Chen R, Yang Y and Jiang J: lncRNA BBOX1-AS1 Aggravates the Development of Ovarian Cancer by Sequestering miR-361-3p to Augment PODXL Expression. Reprod Sci 28 736-744, 2021.

This work is licensed under a Creative Commons Attribution-NonCommercial-NoDerivatives 4.0 International (CC BY-NC-ND 4.0) License. 